\title{
DINÁMICA POBLACIONAL DE INSECTOS COLEÓPTEROS RASTREROS ASOCIADOS AL MARAÑÓN (Anacardium occidentale L.) EN LEÓN, NICARAGUA
}

\section{POPULATION DYNAMIC OF CRAWLING COLEOPTERAN INSECTS FOUND ASSOCIATED TO CASHEW NUT (Anacardium occidentale L.) IN LEÓN, NICARAGUA}

\author{
Jiménez-Martínez Edgardo ${ }^{1}$, Rugama Lovo Ileana ${ }^{2}$, López Vilchez Meyling ${ }^{3}$, Martínez Jorge Gómez ${ }^{4}$ \\ ${ }^{1} \mathrm{PhD}$ en Entomología, Universidad Nacional Agraria, UNA, profesor titular, docente-investigador / edgardo.jimenez@una. \\ edu.ni, Tel: (505) 2263-2609 \\ ${ }^{2-3}$ Ingenieria en protección agrícola y forestal, UNA. \\ ${ }^{4} \mathrm{MSc}$. en agroecología y desarrollo sostenible, UNA, profesor adjunto, docente-investigador.
}

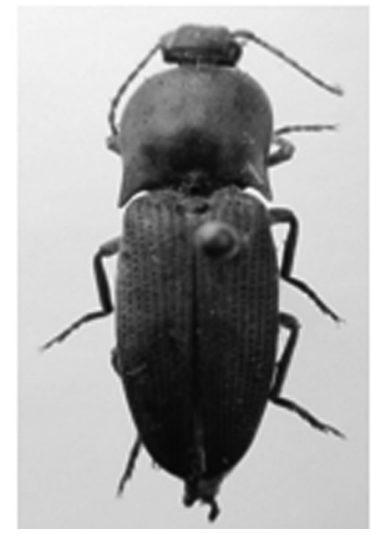

\section{RESUMEN}

El marañón Anacardium occidentale L es originario del noroeste de Brasil, este árbol pertenece a la familia de las Anacardiáceas y al género Anacardium. Actualmente en la zona de occidente de Nicaragua, se han incrementado las plantaciones de marañón manejado bajo dos modalidades orgánicas y convencionales, estas plantaciones se encuentran en manos de pequeños productores los cuales presentan una serie de problemas fitosanitarios. El presente estudio se realizó con el objetivo de identificar y describir el rol de las principales familias del orden coleoptera rastreros asociados al cultivo del marañón orgánico y convencional. Este trabajo se realizó durante el período entre agosto 2009 y marzo del 2010, en la comarca de Chacraseca, del departamento de León, para esto se seleccionaron tres fincas orgánicas y tres fincas convencionales de 0.7 ha cada una, se realizaron muestreos semanales de insectos en cada finca, se seleccionaron cinco sitios por finca, en estos se realizaron capturas manuales de especímenes a través de trampas de caída libre (Pitfall traps). Todos los insectos colectados en el campo se llevaron al laboratorio para su respectiva identificación. Los resultados obtenidos en este estudio fueron los siguientes: de manera general, un mayor número total de insectos en las parcelas de marañón orgánico que en la convencional, se identificaron las principales familias de insectos del orden coleóptera asociados al cultivo del marañón entre ellos: Carabidae, Curculionidae, Scarabaeidae, Tenebrionidae, Se encontró que la familia con mayor número de insectos para ambos sistemas de manejo fue la familia Tenebrionidae y Carabidae. Este es el primer estudio en Nicaragua donde se compara la diversidad de insectos en parcelas de marañón orgánico y convencional, además, esta es la primera vez que se identifican las principales familias, géneros y especies de insectos coleópteros asociados al cultivo de marañón.

Palabras clave: insectos rastreros, marañón, Nicaragua

\section{ABSTRACT}

The cashew (Anacardium occidentale L.), belongs to the Anacardiaceae family and the Anacardium genus and it is native to the northeastern Brazil. Currently in the area of the western Nicaragua, organic and conventional cashew plantations have increased, these plantations are in hands of small producers who have reported a number of phytosanitary problems. This study was carried out to identify and describe the role of the main crawling associated insects in organic and conventional cashew cultivations. This study was performed during the period between August 2009 and March 2010, in the region of Chacraseca, from the department of León, for this study, three organics and three conventional farms were selected, each of 0.7 size for a total of six farms, weekly sampling for insects was performed, five sampling sites were selected per each farm, manual capture of crawling insect specimens was done using Pitfall traps. All insects collected in the field were brought to the laboratory for their respective identification. The results obtained in this study were as follow: a greater number of insects were found in conventional than in organic plots, however, a greater number of families of insects were found in organic than in conventional plots. The main insect families found associated to cashew were as follow: Carabidae, Curculionidae, Scarabaeidae, Tenebrionidae. It was found that the coleopteran families Tenebrionidae y Carabidae were in greater numbers in both studies systems, compared to other families found. This is the first study in Nicaragua where it was compared the diversity of insects in cashew organic and conventional plots, besides, this is the first time that are been reported the main families, genera and species of insects associated to cashew nut.

Key words: terrestrial insect, cashew nut, Nicaragua 
$\mathrm{E}$ 1 marañón (Anacardium occidentale L.) pertenece a la familia de las Anacardiáceas y al género Anacardium y es originario del nordeste de Brasil (Membreño, 2002). Este se cultiva en muchos países tropicales y sub-tropicales como la India, Srilanka, Indochina, Filipinas, Las Antillas, en algunos países de África y en Centroamérica. Los países con mayor producción y área sembrada son: El Salvador y Nicaragua (Gispert et al., 2002, Galdámez, 2004, CIPRES, 2008). El marañón es muy importante por sus cualidades nutricionales ya que es rico en hidratos de carbono, además contiene $45 \%$ de grasa y $20 \%$ de proteínas; al igual aporta altos niveles de fósforo, hierro, calcio, potasio y un alto contenido de vitaminas A, B1, B2 y C (Galdámez, 2004, Durán et al., 2007).

A nivel internacional los mayores productores y exportador de marañón en el mundo son Brasil y la India. El país más demandante es Estados Unidos con una importación anual de 71.5 mil toneladas métricas, que representa aproximadamente el $46.54 \%$ de la importación mundial. El segundo más demandante es la Unión Europea que importa 50 mil toneladas métricas y representan $34.29 \%$ de la importación mundial (MAG, 2002). En el occidente de Nicaragua actualmente el marañón se siembra de forma comercial por pequeños productores de las comunidades de Chacaraseca, Lechecuagos, Las Lomas, El Espino y Larreynaga. En estas comunidades, el cultivo se siembra de manera orgánica y convencional (CIPRES, 2008). Actualmente en esta zona, las plantaciones de marañón orgánico y convencional se han incrementado y se contabilizan unas 500 hectáreas ocupando este rubro el cuarto lugar de importancia seguido del maní, ajonjolí y el maíz. Este rubro es una alternativa para mejorar las condiciones económicas y sociales de las familias campesinas de esta zona, debido a que se está utilizando como materia prima para la industria y la comercialización, además de usarse para la alimentación de animales mayores y menores en época de verano (Silva, 2002).

La agricultura orgánica trata de utilizar al máximo los recursos de la finca haciendo uso de prácticas amigables con el ambiente. Por otro parte, el manejo en las parcelas convencionales, se realiza a través del uso de productos sintéticos basándose en el uso de fuertes cantidades de insumos externos, con un gran consumo energético en forma de agroquímicos y maquinaria, así como el empleo de semillas de alto rendimiento (SIMAS, 1995). Como cualquier otro cultivo, el marañón está expuesto a una gran cantidad de factores limitantes que afectan su desarrollo, reduciendo su rendimiento y desmejorando la calidad de los frutos; entre estos factores se encuentran los de origen biótico, donde se destacan las plagas insectiles como: el chinche patas de hoja (Leptoglossus zonatus), abejita negra (Trigona silvestrianun), entre otros (Galdámez, 2004).
En Nicaragua, los productores de marañón desconocen la diversidad de insectos que están presentes en este cultivo, ya que no existe un reporte formal sobre las principales familias de insectos rastreros asociados al cultivo de marañón, lo que ha contribuido a incrementar los problemas fitosanitarios y de manejo en el cultivo. Debido a esta problemática se realizó este estudio con el propósito de identificar y describir las principales familias de insectos coleópteros asociados al cultivo del marañón, pero sobre todo con el objetivo de conocer más acerca de la diversidad, taxonomía, dinámica e incidencia poblacional de estos insectos asociados al marañón, y de esta manera contribuir con nuevos conocimientos en el desarrollo de mejores estrategias de manejo integrado de plagas en las plantaciones de marañón.

\section{MATERIALES Y MÉTODOS}

Localización geográfica de la zona de estudio. El estudio se realizó en la comarca de Chacraseca del departamento de León, cinco $\mathrm{km}$ al sureste de la cabecera departamental. El municipio está comprendido entre las coordenadas $12^{\circ} 26^{\circ}$ de latitud norte y $86^{\circ} 53^{\circ}$ de longitud oeste, y a una altura de 109.21msnm.

Ubicación de las parcelas experimentales. El estudio se realizó entre los meses de agosto del 2009 a marzo del 2010 en seis fincas de productores, cinco de estas fincas estaban localizadas en la comunidad de Chacraseca y una finca estaba localizada en el kilómetro 85 carretera Managua a León. Las fincas fueron consideradas como las parcelas experimentales; se escogieron tres fincas con manejo orgánico y tres fincas con manejo convencional, Los propietarios de las fincas orgánicas eran los productores Agustín Reyes, Reymundo Mendoza y Melanio Acosta y los productores propietarios de las fincas convencionales eran Orfília Reyes, Gustavo Munguía y William Gurdián.

Clima de la zona de estudio. En la zona donde se realizó el estudio el clima se caracteriza por ser tropical de sabana con pronunciada estación seca entre los meses de noviembre a abril y una estación lluviosa entre los meses de mayo a octubre, con temperaturas promedio de $27^{\circ} \mathrm{C}$ a $29^{\circ} \mathrm{C}$, observándose la más elevada en el meses de marzo y abril y la más baja en los meses de diciembre a enero. La humedad relativa promedio se presenta entre $67 \%$ cuando se registran las mayores temperaturas y $89 \%$ cuando se registran las mayores precipitaciones (ACOPADES, 2010).

Actividad socioeconómica. La base económica de la comunidad es la agricultura; la mayoría de las familias son pequeños y medianos productores, que trabajan la tierra de su propiedad, los principales rubros que se siembran en la zona son el ajonjolí, maíz, marañón, maní, caña de azúcar y yuca; también existe actividad pecuaria, especialmente la crianza de ganado vacuno, cerdos y aves de corral. En la comunidad 
existen 57 productores dedicados a la producción de marañón con un área de siembra de 85 manzanas (MARENA, 2000).

\section{Metodología del monitoreo de insectos rastreros}

Selección de los sitios de muestreo. Se seleccionó la comarca de Chacaraseca porque es una de las zonas del occidente de Nicaragua donde más se cultiva el marañón tanto en fincas con manejo orgánico y convencional. Esta zona presenta buenas condiciones edafo-climàticas para la adaptación del cultivo. Para el establecimiento del ensayo, se seleccionaron seis fincas de productores de marañón, de las cuales tres de ellas eran fincas con manejo orgánico y tres con manejo convencional.

Metodología de muestreo para insectos rastreros. Se seleccionaron tres parcelas orgánicas y tres parcelas convencionales de 0.7 ha cada una. En cada parcela se seleccionaron cinco sitios fijos de muestreo, cada sitio estaba compuesto por un árbol de tres a siete años de edad. Para la captura y colecta de insectos rastreros, se utilizaron trampas de caída libre (Pitfall traps), las cuales consistían en panas plástica de $30 \mathrm{~cm}$ de diámetro, colocadas al ras del suelo con capacidad de cuatro litros de agua, a las cuales se le agregaban cuatro gramos de detergente xedex ${ }^{\circledR}(1 \mathrm{~g} /$ litro de agua), la solución del agua y el detergente se cambiaban semanalmente. Se recolectaron todos los insectos encontrados caídos dentro de las panas, luego se depositaban en viales entomológicos de dos pulgadas de alto y de dos $\mathrm{cm}$ de diámetro, conteniendo alcohol al 75\%, estos se rotularon con la fecha, nombre del productor y el número de sitio muestreado, todos los insectos encontrados en las panas se registraron en una hoja de muestreo.

Procesamiento de las muestras e identificación de insectos. Una vez recolectados los insectos en el campo, se llevaron al laboratorio de entomología y de plagas forestales de la Universidad Nacional Agraria (UNA), posteriormente se extrajeron los insectos de los viales entomológicos y se colocaron en platos petri de $14 \mathrm{~cm}$ de ancho y dos $\mathrm{cm}$ de largo para ser lavados en alcohol al 75\%. Luego cada muestra de insectos se colocó en papel toalla para que se secaran durante 30 minutos a temperatura ambiente. Para la identificación preliminar de los insectos, se utilizó estereoscopio marca ZEISS, Stemi 2000-C, de 10x y marca VANGUARD, de 2 y 4x, para observar las características morfológicas de las familias de insectos, además, se realizó comparación de especímenes con los del museo entomológico de la UNA y se consultó literatura específica sobre taxonomía de insectos. Para el montaje de los insectos en cajas entomológicas, se procedió con la utilización de gradillas entomológicas, pinzas, pinceles, alfileres entomológicos número 4 marca MORPHO de 38 y $45 \mathrm{~mm}$ de longitud, luego cada insecto debidamente etiquetado fue ordenado en las cajas entomológicas.
Variables evaluadas. Número de insectos por familia

Análisis de los datos. Los promedios de cada familia de insecto encontrados en fincas orgánicas y convencionales se compararon por medio de un análisis de t student.

\section{RESULTADOS Y DISCUSIÓN}

Número total de insectos por familias. Se encontraron cuatro familias de insectos asociadas al cultivo de marañon tanto en parcelas organicas como en parcelas convencionales. El mayor numero de insectos en estas familias se presentó en las fincas con manejo orgánico, sin embargo, la familia que presentó el mayor número de insectos fue la familia tenebrionidae con 559 insectos para el sistema orgánico y 419 para el sistema convencional (figura, 1).

Debido a que las parcelas orgánicas no estan sometidas a aplicaciones químicas por parte de los productores, es probable que la diversidad de insectos no sufre un gran impacto. Este es un factor que justífica que se hayan encontrado mayor número de insectos en las parcelas orgánicas que en las convencionales. Por otra parte en las parcelas orgánicas los productores, por lo general, asocian el marañon con otros cultivos como frijol alacin, frijol rojo, frijol blanco, cucurbitaceas, maiz y frutales como cítricos (limón, naranja, mandarina), mangos, aguacate entre otros. Esto ayuda a que haya una mayor diversidad de plantas y de fauna insectil.

Las principales familias, géneros y especies de insectos corresponden a: carabidae (Galerita sp. y Megacephala carolina), curculionidae (varias especies), scarabaeidae ( $\mathrm{Co}$ pris lugubris, Eurysternus sp., Lygirus sp) y Tenebrionidae (Branchus obscurus y Branchus obscurus). Todas las as especias reportadas poseen habito alimenticio masticador.

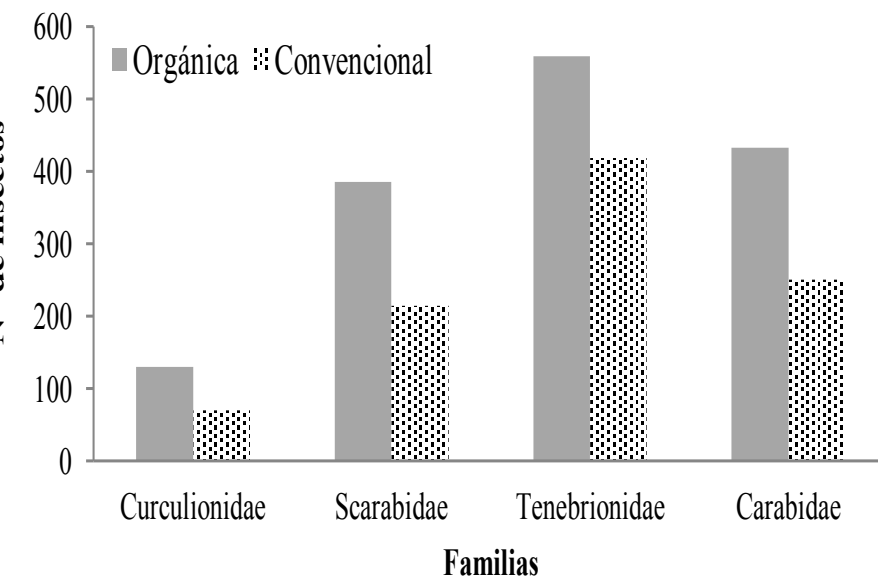

Figura 1. Número total de familias de insectos encontrados en el cultivo de marañón en parcelas orgánicas y convencionales en la comarca Chacraseca, León, agosto del 2009 a marzo del 2010. 
Dinámica poblacional de insectos de la familia Carabidae. Se comparó la fluctuación poblacional de insectos de la familia Carabidae en el cultivo de marañón orgánico y convencional (figura, 2). Estos insectos empezaron a observarse a partir de agosto 28 del 2009 hasta marzo 5 del 2010. Los mayores picos poblacionales fueron observados en tres momentos, en las fechas octubre 16 y noviembre 26 del 2009 y febrero 25 del 2010. En las parcelas de marañón orgánico, las poblaciones de la familia carabidae presentaron su mayor pico poblacional el 26 de noviembre con un total de 42 insectos por trampa. En las parcelas de marañón convencional se observó que el mayor pico poblacional de esta familia se presentó el 25 el febrero con 43 insectos por trampa.

Al realizar la prueba de $\mathrm{t}$ student no se encontraron diferencias significativas (tabla 1). La familia carabidae pertenece al orden coleóptera, son insectos de tamaño pequeño a grande, llegan a medir de dos a $100 \mathrm{~mm}$, cuerpo con forma variable, color oscuro con brillo metálico, generalmente las antenas largas y delgadas se encuentran entre los ojos y las piezas bucales; tienen patas finas y adaptadas para correr, los élitros son rígidos y con estrías. Adultos y larvas son de- predadores activos y voraces durante la noche, también se observan en el día moviéndose rápidamente entre los surcos o sobre las plantas (Sáenz de la Llana, 1990). La literatura menciona que estos insectos por lo general son depredadores, en estas parcelas de marañón se observaron alimentándose de insectos pequeños, lo que confirma que tienen un rol como controladores biológicos en este cultivo.

Dinámica poblacional de insectos de la familia Curculionidae. Se comparó la fluctuación poblacional de insectos de la familia curculionidae en marañón orgánico y convencional (figura, 3). Esta familia se presentó durante todas las fechas de muestreo. Los mayores picos poblacionales se presentaron desde septiembre 11 al 9 de octubre del 2009 y del 16 de diciembre del 2009 al 19 de febrero del 2010. En las parcelas de marañón orgánico, las poblaciones de la familia curculionidae presentaron su mayor pico poblacional los días 16 de diciembre y 29 de enero, con un total de 14 insectos por trampa en cada una de las parcelas. En marañón convencional, se observó que el mayor pico poblacional de esta familia se presentó el 15 de enero y 19 de febrero, con un total de 11 insectos por trampa en cada una de las parcelas.

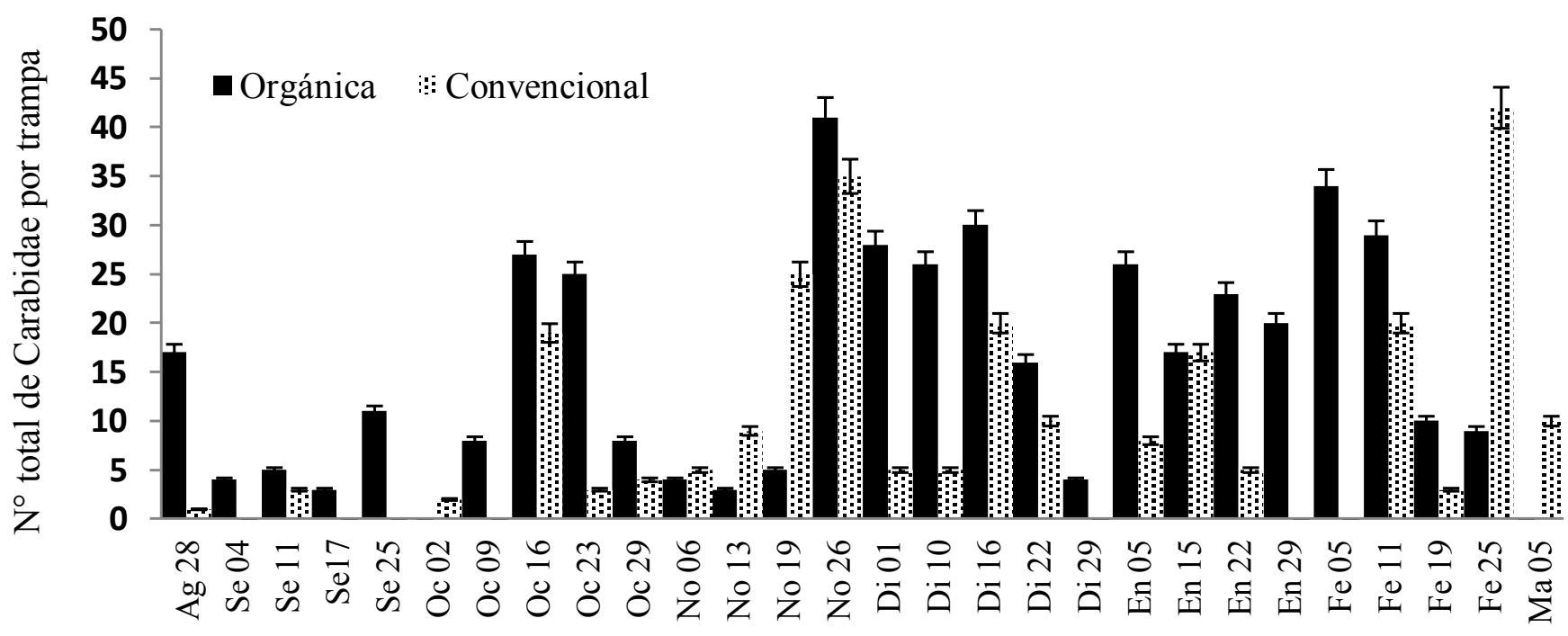

Fechas de muestreos

Figura 2. Dinámica poblacional de insectos de la familia carabidae en el cultivo de marañón orgánico y convencional en Chacraseca, León entre agosto 2009 y marzo 2010. 


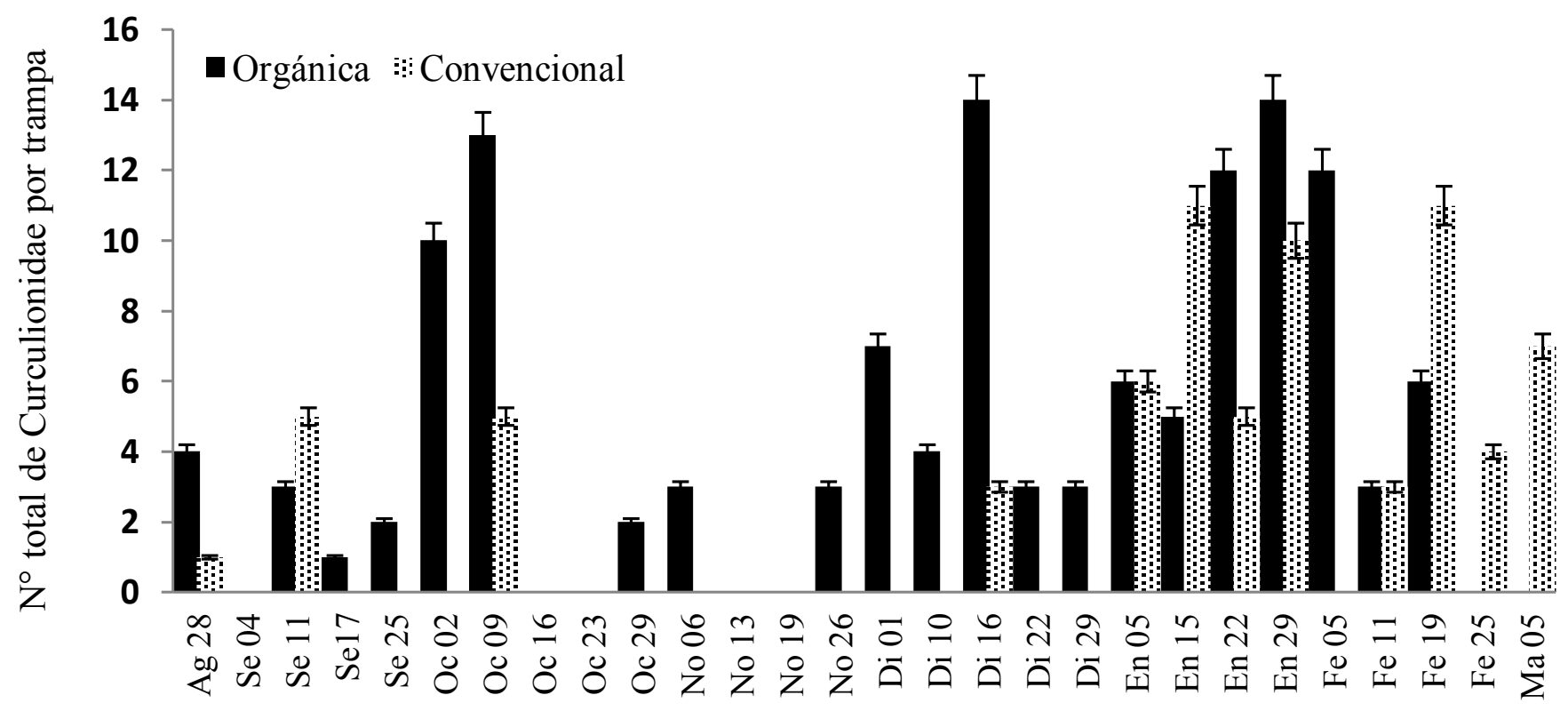

\section{Fechas de muestreos}

Figura 3. Dinámica poblacional de insectos de la familia curculionidae en el cultivo de marañón orgánico y convencional en Chacraseca, León entre agosto 2009 y marzo 2010.

Al realizar el análisis de t Student se encontró diferencias significativas $(\mathrm{P}=0.046)$. El menor número de estos insectos lo presentó en marañón convencional (4.78 insectos por trampa), comparado con parcelas orgánica que registró un promedio de 6.29 insectos por trampa (tabla 1). La familia Curculionidae pertenece al orden coleóptera, son insectos de tamaño pequeño a grande, llegan a medir entre uno y 35 $\mathrm{mm}$ de longitud, cuerpo con forma variable generalmente alargada a ovalada, la coloración es muy variada, estos insectos son conocidos como picudos porque su cabeza se prolonga hacia adelante formando una especie de trompa, su aparato bucal es masticador, se alimentan de cualquier parte de la planta y perforan frutos y nueces, son considerados plagas de importancia agrícola (CATIE, 1991). Estos tipos de insectos son reportados por los productores como insectos que ocasionalmente se alimentan del fruto y el falso fruto del marañón.

\section{Dinámica poblacional de insectos de la familia Scarabaei-}

dae. Se comparó la fluctuación poblacional de insectos de la familia scarabaeidae en el cultivo de marañón orgánico y convencional (figura, 4). Esta familia se presentó durante todas las fechas de muestreo, desde el 28 de agosto del 2009 al cinco de marzo del 2010. Los mayores picos poblacionales se dieron durante el 25 de septiembre, del 19 de noviembre al 22 de diciembre del 2009 y del 15 de enero al 19 de febrero del 2010. En las parcelas de marañón orgánico se encontró que las poblaciones de la familia scarabaeidae se presentaron desde la primera fecha 28 de agosto ocurriendo su mayor pico poblacional el 22 de diciembre, con un total de 34 insectos por trampa, en las parcelas de marañón convencional, las poblaciones de insectos se presentaron a partir del 4 de septiembre, ocurriendo su mayor pico poblacional el 25 de septiembre, con 47 insectos por trampa.

Al realizar el análisis de t Student se encontró diferencia significativa $(\mathrm{P}=0.01)$, el menor número de insectos lo presentó la parcela convencional con un promedio de 5.83 insectos por trampa, comparado con la parcela orgánica que presentó un promedio de 9.64 insectos por trampa (tabla, 1). La familia scarabaeidae pertenece al orden coleóptera, llegan a medir de tres a $180 \mathrm{~mm}$ de longitud, es una de las familias más grandes y tienen gran variedad de tamaño, colores y hábitos, son conocidos como ronrones o chocorrones, Los machos de algunas especies presentan cuernos, tanto en la cabeza como en el pronoto, su aparato bucal es masticador se alimentan de las raíces de las plantas causando un pobre desarrollo (Sáenz de la Llana, 1990; Jiménez-Martínez. 2009). En el caso del marañón, estos insectos se encontraron siempre presente en las trampas, lo que nos indica que buen número de estos insectos son atraídos por el marañón, debido al hábito nocturno de los adultos y a su vuelo errático, casi siempre caían en las trampas, no hay reportes de los productores de que estos insectos son plagas del marañón. 


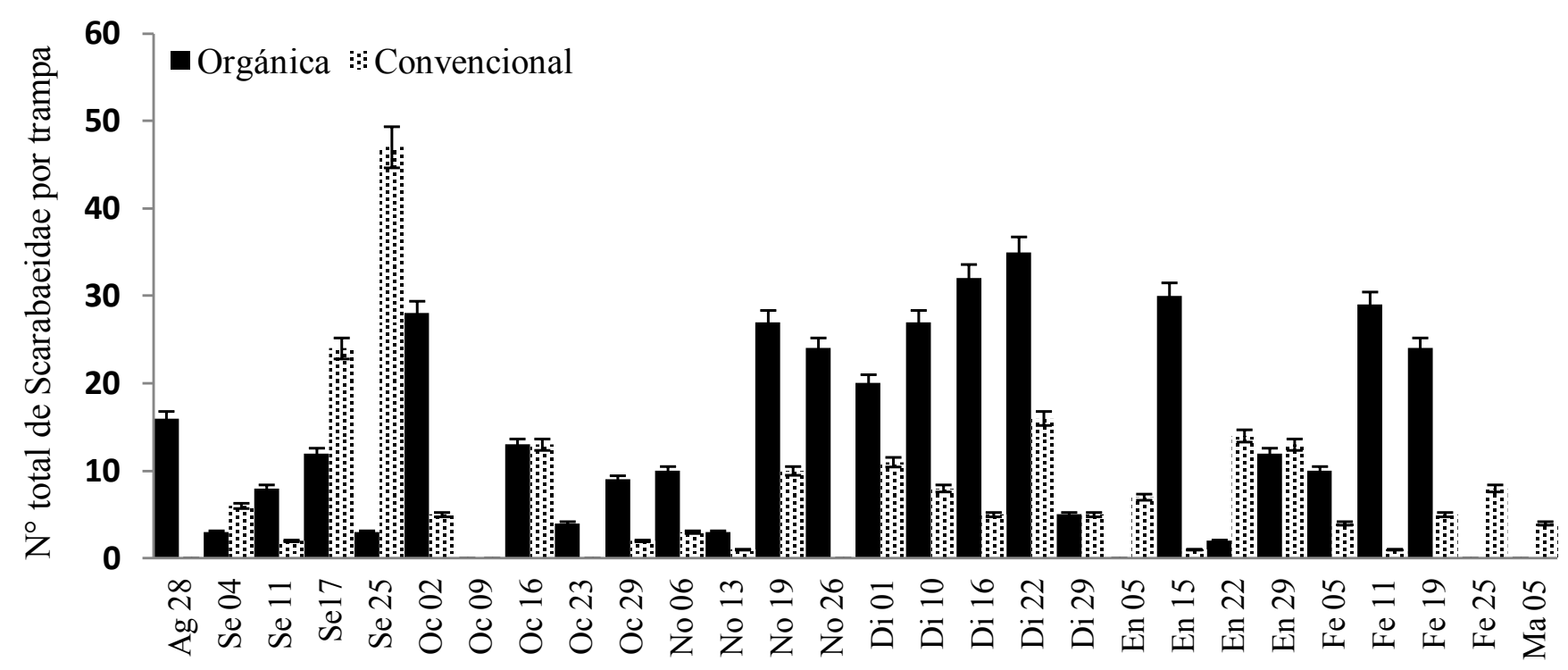

Fechas de muestreos

Figura 4. Dinámica poblacional de insectos de la familia scarabaeidae en el cultivo de marañón orgánico y convencional en Chacraseca, León entre agosto 2009 y marzo 2010.

Dinámica poblacional de insectos de la familia Tenebrionidae. Se comparó la fluctuación poblacional de insectos de la familia tenebrionidae en el cultivo de marañón orgánico y convencional (figura, 5). Estos insectos se presentaron a partir de la primera fecha de muestreo, el 28 de agosto del 2009 al 5 de marzo del 2010. Estos insectos fueron encontrados en todas las fechas de muestreo tanto en las parcelas orgánicas como en las convencionales. Los mayores picos poblacionales se registrarón entre las fechas noviembre 19 del 2009 a febrero 19 del 2010. En las parcelas de marañón orgánico las poblaciones de insectos se presentaron a partir de la fecha 11 de septiembre ocurriendo su mayor pico poblacional el 5 de febrero, con un total de 45 insectos por trampa. En las parcelas de marañón convencional las poblaciones de insectos se presentaron a partir de la fecha 28 de agosto, ocurriendo su mayor pico poblacional en la fecha 29 de enero con 48 insectos por trampa.
Al realizar el análisis de t student no se encontraron diferencias significativas (tabla 1). La familia Tenebrionidae pertenece al orden Coleóptera, son de tamaño pequeño a grande, miden de dos a $65 \mathrm{~mm}$, presentan una coloración oscura, casi siempre pardo o negro y sus ojos en forma "C" tienen el pronoto más ancho que la cabeza está compuesta por un grupo de insectos morfológicamente muy heterogéneos. El hábito alimenticio es muy variado suelen alimentarse de raíces, follaje y plántulas, son considerados plagas de algunos cultivos agrícolas (CATIE, 1991; Jiménez-Martínez, 2009). Estos insectos son considerados plagas de las raíces, del cultivo del marañón, no se observó alimentándose de follaje, pero si se observó en gran número en las trampas, lo que nos hace suponer que son insectos que viven y se reproducen exitosamente en el cultivo del marañón. 


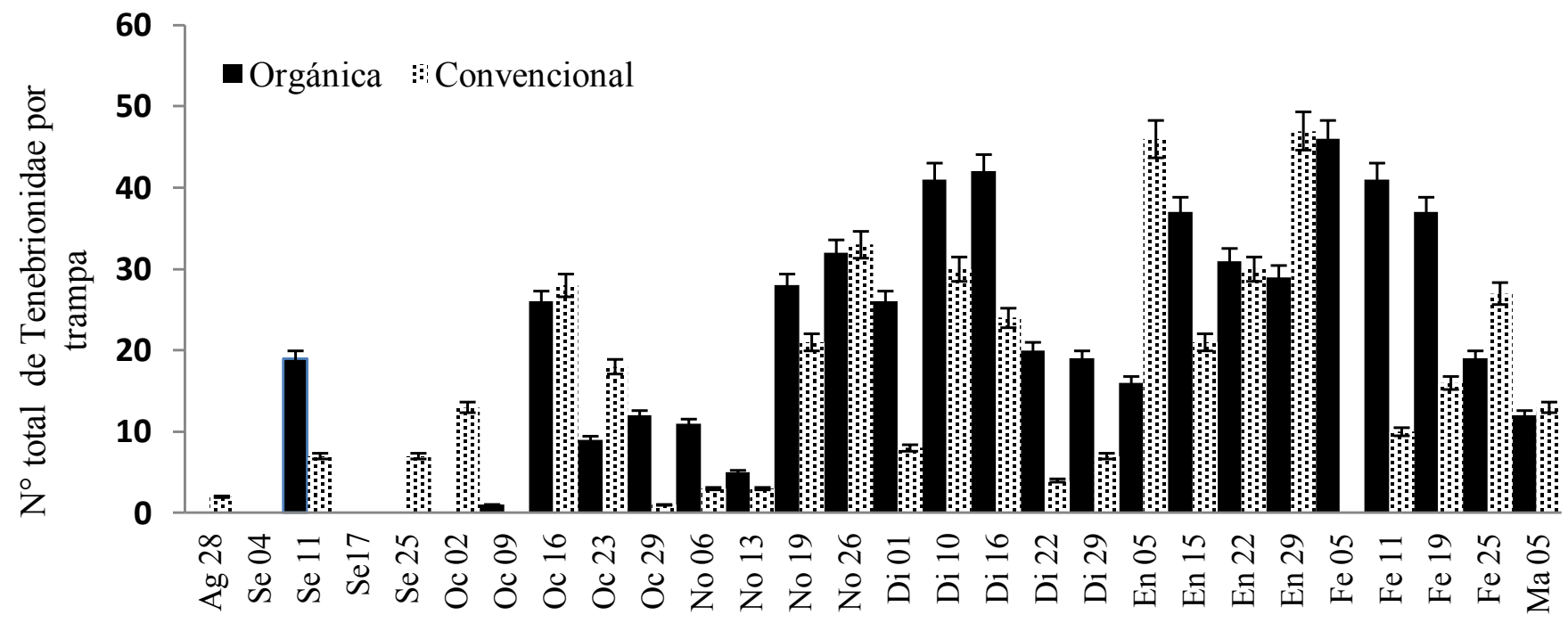

Fechas de muestreos

Figura 5. Dinámica poblacional de insectos de la familia tenebrionidae en el cultivo de marañón orgánico y convencional en Chacraseca, León entre agosto 2009 y marzo 2010.

Tabla 1. Análisis de varianza de las fluctuaciones poblacionales de las familias carabidae, curculionidae, scarabaeidae y tenebrionidae encontrados en el cultivo de marañón orgánico y convencional, en Chacraseca, León entre los meses agosto 2009 y marzo 2010

\begin{tabular}{|c|c|c|c|c|}
\hline Variable & Carabidae & Curculionidae & Scarabaeidae & Tenebrionidae \\
\hline Tratamiento & $*$ Media \pm SE & $*$ Media \pm SE & $*$ Media \pm SE & $*$ Media \pm SE \\
\hline Orgánico & $8.43 \pm 1.08$ & $6.29 \pm 1.95$ & $9.64 \pm 1.06$ & $11.17 \pm 1.48$ \\
\hline Convencional & $8.42 \pm 1.50$ & $4.78 \pm 1.08$ & $5.83 \pm 0.91 \quad b$ & $10.26 \pm 1.84$ \\
\hline $\mathrm{CV}$ & 95.02 & 146.74 & 73.84 & 93.90 \\
\hline $\mathrm{P}$ & 0.99 (NS) & 0.0468 ( DS) & $0.01(\mathrm{DS})$ & $0.70(\mathrm{NS})$ \\
\hline $\mathrm{F} ; \mathrm{df} ; \mathrm{n}$ & $0.00 ; 79 ; 81$ & $0.53 ; 59 ; 61$ & $7.01 ; 65 ; 67$ & $0.15 ; 73 ; 75$ \\
\hline
\end{tabular}

ES: Error estándar, n: Número de datos usados, DS: Diferencia significativa, F: Fisher calculado, Df: Grado de libertad, CV: Coeficiente de variación, P: Probabilidad

* Medias con letras distintas existe diferencia significativas.

\section{CONCLUSIONES}

Se reporta que las principales familias de insectos del orden coleóptera asociadas al marañón son carabidae, curculionidae, scarabaeidae y tenebrionidae.

Se identificaron y se describieron las dinámicas poblacionales de insectos asociados al marañón entre estos los miembros de las familias carabidae, curculionidae, scarabaeidae y tenebrionidae, encontrándose, que estas familias mantuvieron su presencia durante todo el período de muestreo en el cultivo tanto en la época de crecimiento vegetativo como en la época de floración y fructificación del cultivo del marañón.

\section{AGRADECIMIENTO}

Los autores agradecen a la cooperación Sueca a través de la agencia internacional para el desarrollo ASDI, por haber proveído los fondos para esta investigación, a la Universidad Nacional Agraria por brindarnos el apoyo logístico para el desarrollo de este estudio, así como también agradecemos a los productores de marañón de Chacraseca, Leon. Se extiende un especial agradecimiento a los compañeros de trabajo señor Alex Cerrato y Oswaldo Rodríguez del museo entomológico de la UNA, ellos contribuyeron en gran manera con el montaje e identificación taxonómica de los insectos reportados. 


\section{REFERENCIAS BIBLIOGRÁFICAS}

ACOPADES (Asociación de Comité Pastoral para el Desarrollo Social de la Comarca de Chacaraseca). 2010. Información sobre

Chacaraseca, León. Consultado 23 ene. 2011. Disponible en http://acopadeschacraseca.blogspot.com/.

Andrews, K; Caballero, R. 1989. Guía para el estudio de órdenes y familias de insectos de Centroamérica. Escuela Agrícola Panamericana, Zamorano, Honduras. 179 p.

CIPRES (Centro para la Promoción, la Investigación y el Desarrollo Rural y Social). 2008. Guía para el manejo de las principales plagas de marañón orgánico en Nicaragua. p. 39.

CATIE (Centro Agronómico Tropical de Investigación y Enseñanza). 1991. Plagas y enfermedades forestales en América Central, guía de campo. Turrialba, CR. p. 90.

Durán, RF; Moncada, E; Rodríguez, GA; Rey, AM. 2007. Manual curativo con frutas y plantas medicinales. Ediciones Grupo Latinos, Bogotá, Colombia. $680 \mathrm{p}$.

Galdámez, A. 2004. MAG (Ministerio de Agricultura). Guía técnica del cultivo del marañón en El Salvador. Programa nacional de frutas de El Salvador. p. 69.

Gispert, C; José, G; Josué, FG. 2002. Enciclopedia práctica de la agricultura y la ganadería. Grupo editorial Océano, Barcelona, España. 1028 p.

Jiménez-Martínez, E. 2009. Entomología. UNA (Universidad Nacional Agraria). Managua. NI. 111 p.

MAG (Ministerio de Agricultura y Ganadería). 2002. Boletín mercado de la nuez del marañón. Consultado 13 ene. 2010. Disponible en http://www1.minambiente.gov.co/viceministerios/ambiente/mercados_verdes/INFO\%20SECTORIAL/Boletin\%20de\%20 Mercado\%20de\%20Mara\%F1on.pdf.

MARENA (Ministerio del Ambiente y los Recursos Naturales). 2000. Planes ambientales municipales del departamento de León, municipio de León. 37 p.

Membreño, P. 2002. El Marañón (Anacardium occidentales, L). p. 28.

Sáenz, M; De La Llana, A. 1990. Entomología sistemática. UNA (Universidad Nacional Agraria). Managua, NI. 225 p.

Silva, G. 2002. Insecticidas vegetales una vieja y nueva alternativa para el manejo de plagas. CATIE. Manejo integrado de plagas y Agroecología. Turrialba, CR. p. 60.

SIMAS (Servicios de Información Mesoamericana sobre Agricultura). 1995. Agricultura orgánica. p. 148. 